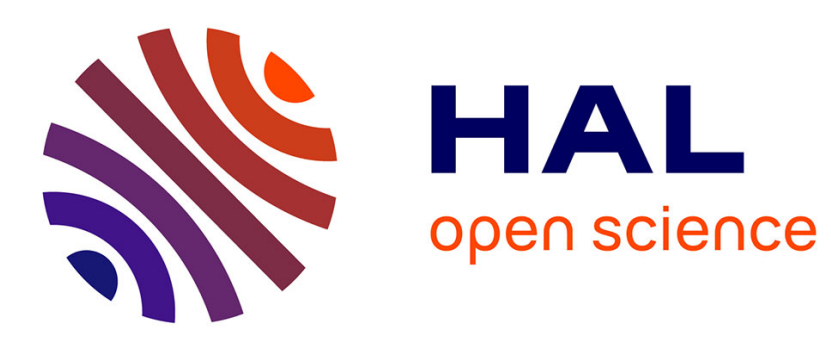

\title{
Traduction automatique des langues et modélisations des interactions langagières
}

Agnès Salinas

\section{To cite this version:}

Agnès Salinas. Traduction automatique des langues et modélisations des interactions langagières. Langages, 2001, Psycholinguistique et intelligence artificielle, 35 (144), pp.99 - 123. 10.3406/lgge.2001.901 . hal-01917313

HAL Id: hal-01917313

https://hal-normandie-univ.archives-ouvertes.fr/hal-01917313

Submitted on 9 Nov 2018

HAL is a multi-disciplinary open access archive for the deposit and dissemination of scientific research documents, whether they are published or not. The documents may come from teaching and research institutions in France or abroad, or from public or private research centers.
L'archive ouverte pluridisciplinaire HAL, est destinée au dépôt et à la diffusion de documents scientifiques de niveau recherche, publiés ou non, émanant des établissements d'enseignement et de recherche français ou étrangers, des laboratoires publics ou privés. 


\title{
Traduction automatique des langues et modélisations des interactions langagières
}

MME Agnès Salinas

\begin{abstract}
This paper aims to tackle the question of automatic translation of languages, from a psycholinguistic viewpoint. We shall consider the issue in relation to the following problematic areas: intra- and extra-linguistic interactions between dialogues and, more particularly, interactions between cultural substratum's. An ALT (Automatic Language Translation) system should be able to pick out the connections between what is said, by whom and to whom, and incorporate the cultural and developmental variables. The works presented here illustrate the complexity involved in constructing a method for the exchange of information between source language and target language, especially when the formatting can differ in many ways, for example in spatial and contextual dimensions. In fact, one cannot effectively use computers to study dialogues without first analysing the cognitive complexity of the representations generated by individual cultural and linguistic substratum's.
\end{abstract}

\section{Citer ce document / Cite this document :}

Salinas Agnès. Traduction automatique des langues et modélisations des interactions langagières. In: Langages, $35^{\mathrm{e}}$ année, n¹44, 2001. Psycholinguistique et intelligence artificielle. pp. 99-123;

doi : https://doi.org/10.3406/lgge.2001.901

https://www.persee.fr/doc/lgge_0458-726x_2001_num_35_144_901

Fichier pdf généré le 03/05/2018 
Agnès SALINAS

Université de Caen, MRSH

LPCP, ModeSCoS

\section{TRADUCTION AUTOMATIQUE DES LANGUES ET MODÉLISATIONS DES INTERACTIONS LANGAGIËRES}

L'objectif est de présenter un éclairage psycholinguistique sur la question de la traduction automatique des langues en prenant en compte la problématique des interactions intra et extralangagières dans le dialogue et notamment les problèmes liés aux substrats culturels. La communication précédente montre qu'il n'est pas évident pour un système d'identifier ce qui est dit, d'interpréter pourquoi cela est dit et de déterminer quoi dire en réponse en faisant preuve d'un certain degré d'adaptabilité à l'utilisateur. Nous ajoutons à ces considérations que le système devrait aussi repérer le rapport entre ce qui est dit, qui le dit, à qui et ceci en tenant compte de variables culturelles et développementale. Plus précisément, cette approche psycholinguistique souligne la complexité de la construction de l'échange en langue cible lorsque le formatage de la communication est conçu différemment selon des variables telles que les dimensions spatiales et contextuelles.

On ne peut, en effet, étudier le dialogue par le biais d'une machine sans analyser la complexité cognitive des représentations engendrées par le substrat culturel et linguistique de l'individu. Il est déjà difficile de l'établir au sein d'une même langue puisque les individus, la plupart du temps, vivent et expérimentent différemment le contenu des propositions langagières porteuses de significations personnelles; dès lors, si l'on ajoute une variation culturelle à la variable interindividuelle, les champs d'intercompréhension en sont encore modifiés. Peut-on cependant tenter d'analyser des formats courants d'interactions, des tendances interactives dans lesquels les sujets psychologiques peuvent se reconnaître? C'est ce que nous allons proposer avec une analyse de dialogues interculturels qui s'éloignent des formats de nos dialogues monoculturels occidentaux. Nous postulons que ce genre d'analyse psycholinguistique peut guider les travaux de modélisation en Traduction Automatique des Langues. 


\section{Problématique : les niveaux de catégorisations linguistiques et de formats pragmatico-sémantiques à prendre en compte pour une Traduction Automatique des Langues}

\subsection{Quid de la communication et de l'intercompréhension dans la confrontation $L 1 / L 2$ ?}

Pour qu'il y ait intercompréhension dans le dialogue, et surtout dans le dialogue retranscrit en $\mathrm{L} 2$, il faut qu'il y ait construction d'un terrain commun, lequel doit rassembler des codes cognitifs, linguistiques et communicatifs communs aux deux interlocuteurs. En communication exolingue avec un natif français, un sujet apprenant construira une interlangue avant de s'approprier définitivement le français en L2. Au sein de celle-ci, des interférences renseignent le psycholinguiste sur les avancées cognitives, langagières et sur l'appropriation des nouveaux codes d'interlocution. Sans cette progression, on ne peut parler que de bilinguisme relatif avec une simple retranscription littérale des catégories linguistiques et des codes de communication de la langue source. C'est avant tout le sujet psychologique, le sujet parlant et l'usage qu'il fait de sa deuxième langue qui seront ici analysés, avec pour objectif une adéquation des attentes que l'on peut avoir raisonnablement en fonction des langues en présence.

\subsection{Inscription du contexte et du spatial dans les formats culturels de référenciations}

L'espace vécu, ressenti, exprimé est à la base de la psychologie topologique et le fonctionnement interactif de la communication dépend de cet espace psychologique. Lewin, psychosociologue fondateur du Centre de Recherche sur la Dynamique des Groupes en 1945, a posé les principes de cette psychologie spatiale: il étudie le comportement de l'individu par rapport à la configuration générale de son espace de vie ou de son champ psychologique. Le champ psychologique d'un sujet humain est l'organisation, la configuration des significations que les choses, les êtres ou les significations ont pour lui. Il y aurait par conséquent un espace significatif, d'où une psychologie topologique. Suite à ces travaux fondamentaux pour la dynamique des groupes primaires avec une explicitation de la réalité spatiale et socio-affective qui en constitue les bases, la gestion de ce champ psychologique inter-individuel facilite la prise en charge d'un groupe ainsi que la compréhension des phénomènes qui s'y déroulent. Dans la continuité de ces travaux, l'École de Palo Alto se situe au niveau de cette psychologie topologique, et Hall (1972) parle de proxémie pour définir plus précisément ce champ psychologique. Il y aurait donc une 
dimension culturelle qui séparerait les individus entre eux et cet espace significatif ou champ psychologique serait tout à fait mesurable. Il permettrait de signifier d'emblée le type de communication interpersonnelle dont il serait question: communication intime, personnelle ou publique selon la distance entre les personnes (distance qui varie selon la culture d'appartenance). Des malentendus, des oppositions, des préjugés peuvent naître du non-respect de cette dimension spatiale culturelle cachée ou non consciente. C'est à partir de cette psychologie topologique, définie par des auteurs tel Hall (1972), que nous comparerons des groupes culturels qui n'ont pas la même gestion topologique de la communication et que nous aborderons la problématique de la traduction des langues.

Ainsi nous avons comparé le processus d'apprentissage d'une seconde langue par rapport à ces référenciations culturelles et c'est au cours de ces travaux que nous avons élaboré une typologie psycholinguistique des langues qui prend en compte d'une part, les rapports à l'espace qu'on identifiera en psycholinguistique ou en linguistique sous le terme de deixis ( $c f$. Morel, 1990), et d'autre part, l'inclusion du contexte et de ces facteurs spatiaux dans la langue du groupe culturel d'appartenance.

\subsubsection{Typologie psycholinguistique des langues comme outil théorique et méthodologique}

Dans les langues à tendance implicite, le contexte, le monde environnant, l'espace sont pris en compte de manière active et implicite dans la référenciation, alors que dans des langues explicites, l'individu est le vecteur essentiel de la référenciation, l'espace-temps n'intervenant que de façon complémentaire. Il y a de ce fait une certaine indépendance des énoncés par rapport au contexte. La référenciation départageant de manière explicite la deixis entre moi et ici-maintenant de façon grammaticale et pré-réglée (hors de la présence des interlocuteurs, on peut comprendre la référenciation évoquée lors d'un dialogue en langue explicite, ce qui n'est pas toujours possible en langue implicite).

Exemple téléphonique:

- en français, $X$ se présente par son nom, et s'adresse à $Y$, autre nom de référence en langue explicite (personnalisation pronominale ou nominale);

- en cambodgien, langue implicite et dépendante du contexte spatial et environnemental, l'on ne dit pas son nom, mais l'on désigne si nécessaire le lieu d'où l'on parle vers le lieu vers lequel l'on s'adresse pour échanger (personnalisation spatiale).

Lorsqu'on ignore ce mode de catégorisation ou de référenciation, l'échange peut sembler incompréhensible. 
La typologie différentielle des langues que nous avons élaborée ( $c f$. Salinas 1994, 1996, 1998) permet de rendre compte de façon plus fine de ces divergences culturelles et linguistiques. Ainsi, en tant que psychologues, nous relevons des éléments culturels et pragma-linguistiques dans les langues concernées; nous les utilisons pour différencier le parcours d'acquisition de la langue seconde. Cette typologie propose non pas seulement de retranscrire la difficulté linguistique de l'apprentissage d'une seconde langue, mais aussi de retracer le mode d'interaction en langue 1 et en langue 2, la forme catégorielle et la fonction de communication, l'usage de la langue tel que le sujet a l'habitude de la pratiquer.

Sur un continuum de langues, nous proposons ainsi deux pôles opposés: un pôle implicite, avec des langues dépendantes du contexte et à référentiels non marqués, comme le cambodgien (avec des mots invariants, l'utilisation préférentielle des classifications sémantiques, une deixis spatiale pour l'évocation du Soi et des précisions post-posées au cours de l'échange avec un énoncé initial très imprécis, à construire de façon externe avec autrui) et un pôle explicite avec des langues comme le français ou l'espagnol (avec des mots mouvants, des catégories grammaticales importantes, une deixis égocentrique "moi-ici-maintenant», interne, des pré-déterminations linguistiques dans et dès les premiers échanges). Un grand nombre de langues peuvent donc être situées par le psycholinguiste vers le pôle implicite ou vers le pôle explicite: c'est le passage d'un pôle à l'autre qui représentera le plus grand degré de difficulté dans un processus de traduction ou dans le processus d'acquisition de la seconde langue. Selon cette approche interculturelle et pragma-linguistique, le passage d'une langue 1 à une langue 2 de même pôle facilitera au contraire la traduction ou l'acquisition.

Pôle implicite continuum de langues Pôle explicite

Langues dépendantes du contexte

Exemple: cambodgien

Catégories linguistiques:

1) Invariance des mots

Genre et nombre ne sont pas marqués.

2) Catégorisations sémantiques

avant tout

3) Langues lococentriques

(deixis spatiale)

Communication:

4) Peu de précisions initiales
Langues indépendantes du contexte

Exemples: espagnol, français

\section{1) Mouvance des mots \\ Genre et nombre sont marqués}

2) Catégories grammaticales

3) Langues égocentriques

(deixis moi-ici-maintenant)

4) Pré-déterminations 
5) Ordre syntaxique important ;

peu de connecteurs

Soi dans l'interaction:

6) Personnalisation secondaire, spatiale

Pas d'utilisation de pronom personnel.
5) Connecteurs importants

6) Personnalisation primaire

Figure 1: Typologie psycholinguistique des langues (1994).

Selon le type de langues en présence et selon les variations de routines interactives culturelles, la référenciation interpersonnelle (étudiée par l'utilisation correcte de catégories linguistiques telles que les pronoms personnels, les connecteurs, les anaphores) sera plus ou moins bien adaptée en langue seconde.

\subsection{Utilisation ou non du code-switching dans les premières traductions et implications sur les automatismes de communication}

\subsubsection{Le code-switching comme stratégie d'apprenant et comme jonction des catégorisations entre $L 1$ et $L 2$}

Au travers de travaux antérieurs (Salinas, 1994, 1996, 1998), et à la suite de Cammarota et Giacobbe, 1986, nous avons montré qu'il y avait une fonction du code-switching (ou alternance codique entre L1 et L2): les ponts entre L1 et L2 s'entrevoient dans le code-switching et la création d'une interlangue permet un premier recul métalinguistique. Ce recul ainsi que les possibilités d'expression orale facilitent l'acquisition de la L2. Le code-switching serait donc bénéfique puisqu'il autoriserait une véritable stratégie d'apprenant. Lorsqu'une langue implicite telle que le cambodgien ne peut pas servir de substrat, ni fournir d'hypothèses par rapport à la L2 (français), il n'y a pas de possibilité de code-switching. Que se passe-t-il dans ce cas? On s'aperçoit que le contenu est français mais grammaticalement incorrect et qu'il véhicule des catégorisations linguistiques en L1. Il y aurait donc une différenciation culturelle: on peut parler, dans les travaux que nous avons réalisés, d'une importance de la forme catégorielle proche autorisant le code-switching pour les enfants hispanophones, ce qui facilite le processus d'acquisition et pour les enfants khmérophones, d'une inhibition catégorielle qui empêche l'utilisation du code-switching et inhibe l'expression de la forme et du contenu catégoriels en langue française. Nous allons en citer quelques exemples ciaprès. 


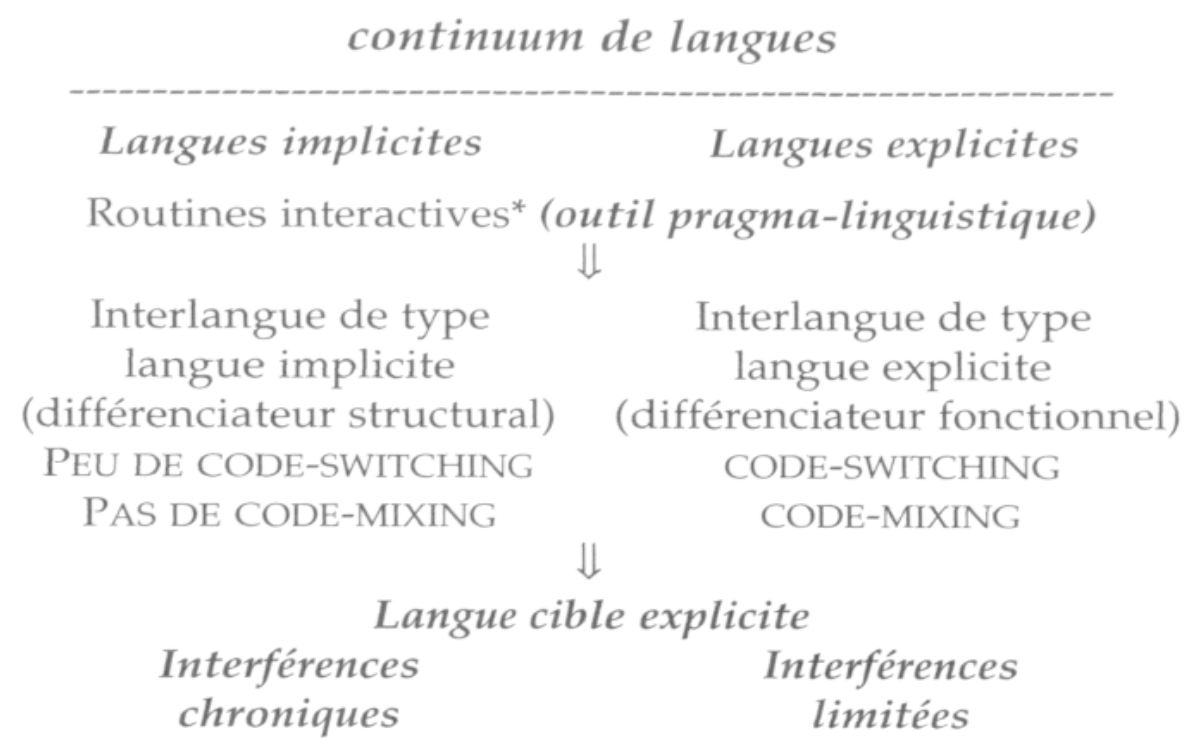

Figure 2: Modèle de répertoire de langues pour l'acquisition de la L2 (outil psycholinguistique théorique : typologie différentielle).

\subsubsection{Automatismes de communication et implications}

Le code-switching autorise la correction du français/L2 par le natif et permet à l'apprenant de repérer les grandes différences entre L1 et L2 au niveau des catégories linguistiques et de l'usage linguistique. Lorsque langues 1 et 2 appartiennent au même type de langue, le code-switching favorise ainsi des mises en correspondance (ou en divergence) et l'établissement $d$ 'hypothèses métalinguistiques qui vont être corrigées par le natif. Le code-switching aboutit ainsi à une possibilité de stratégie d'apprenant.

Ainsi, les mêmes types de langues permettent aussi d'éviter des erreurs graves dans la communication puisqu'on y retrouve les mêmes grands principes de codification du message ainsi que les mêmes automatismes de formes de communication.

\subsubsection{La fonction de l'interlangue}

L'interlangue peut être considérée en soi comme une troisième langue qui sert la transformation des catégorisations; on la retrouve dans nos résultats, sous forme de code-switching chez les hispanophones acquérant le français. Chez les Cambodgiens, seul le français est utilisé puisque le mélange des catégories linguistiques est difficile (trop différent) : c'est ce français approximatif qui est en lui-même une troisième langue car il porte la trace des catégories cambodgiennes. Dans le rapport exolingue avec un natif, il serait intéressant de vérifier les corrections et les reformulations qu'il effectue à l'égard d'un apprenant qui alterne L1 et L2, alors qu'il est peut-être plus difficile, pour ce même natif, de corriger chez l'apprenant une structure en français, mais catégorisée en cambodgien. 
C'est la procédure de transformation catégorielle qui n'est pas de même nature: l'hispanophone peut revenir quand il le souhaite à son interlangue et confronter ses hypothèses d'apprenant (à condition qu'il ait acquis ce niveau métalinguistique, condition d'une bonne expression bilingue); le cambodgianophone ne possède pas de passerelle d'interlangue et peut subir un refus global de la part du natif (l'énoncé pouvant être complètement incompréhensible).

Pour étudier l'utilisation du français comme seconde langue, il est important de tenir compte de la langue source, mais aussi de son fonctionnement interactif, socio-cognitif et culturel. Dans le cadre du bilinguisme cambodgien/français, des indices linguistiques tels que l'utilisation de pronoms personnels, de connecteurs, de reprise anaphorique, de l'ordre syntaxique en français L2, ne peuvent être étudiés sans une approche interculturelle, psycholinguistique et dialogique: l'économie de cette approche risquerait d'entraîner une interprétation erronée des indices catégoriels. En effet, chaque langue porte en elle sa vision du monde; elle est le reflet d'une catégorisation culturelle; apprendre une seconde langue, c'est donc apprendre à comparer. C'est aussi apprendre à découper différemment le monde, avec d'autres mots. Chaque langue exprime des réseaux de conventions culturelles non dites qui règlent les échanges, appris lors des routines interactives. Si ces langues sont interprétantes de réseaux très différents, dès lors que se passe-t-il dans les cas de bilinguisme? Il se peut que certains types de langues très différenciés entravent le recul métalinguistique et les ponts catégoriels lorsque l'apprentissage est amorcé.

\subsubsection{Style cognitif et expérience des langues}

Tout ce qui constitue l'expérience langagière d'un individu sera ainsi marqué selon les rapports entre les langues qu'il a l'occasion d'apprendre et de pratiquer. En psychologie différentielle, on s'arrête précisément à tout ce qui constitue la singularité d'un style cognitif et langagier. Dès lors nous considérons que la définition d'un style cognitif doit prendre en compte cette expérience langagière. Ainsi, dans le parcours d'acquisition du langage au sein d'une même culture, l'individu réagit quotidiennement avec des pratiques langagières de tendance externe ou interne et la confrontation de ces normes internes et externes de "savoir-dire" et "comment le dire " est à l'origine de nombreux malentendus et l'exclusion sociale y prend souvent sa source. Mieux connaître les fonctionnements linguistiques à tendances externes ou internes permettrait sans doute de limiter les dysfonctionnements dialogiques.

Apprendre une seconde langue, c'est apprendre des normes d'interactions culturelles, c'est aussi apprendre à découper le monde différemment : 
il faut donc intérioriser de nouvelles catégorisations dans les formats d'énonciation ( $c f$. notion de catégories, de scenarii et de scripts d'Abelson, 1976). L'interlangue peut être considérée par le psycholinguiste comme une troisième langue qui sert la transformation des catégorisations; on la retrouve sous forme de code-switching chez les hispanophones acquérant le français. Nous retrouvons ces difficultés à un niveau plus moléculaire lorsque les codes langagiers externes sont confrontés à des codes langagiers internes (au niveau subculturel au sein d'une même culture): les échanges ne se situent plus sur le même plan et les antagonismes sociaux surgissent. Des retombées pratiques de ces recherches s'effectuent au niveau d'une meilleure connaissance des sujets migrants, mais aussi des sujets monolingues illettrés ou dits "bas niveau de qualification", très dépendants du contexte, de l'externalité, ainsi que du champ proxémique (psychologie topologique).

\subsubsection{Les types de langues et la T.A.L.}

En résumé, le sujet dont la L1 se situe dans le même pôle que la L2 conserve le bénéfice de codes linguistiques, cognitifs, spatiaux, communicationnels similaires et ne devrait pas être inhibé au niveau de l'acquisition de sa L2, à l'inverse d'un sujet qui devrait passer d'une L1 d'un pôle implicite, comme le cambodgien ou le malgache, à une L2 comme le français ou le portugais. Ses automatismes de traduction seraient bouleversés, dans ce dernier cas de figure, et lui demanderaient un effort particulier d'adaptation. Cette adaptation n'est pas aisée si l'on en croit les difficultés que rencontrent les populations de ce type (Coréens en Amérique du Sud; Laotiens en Guyane, Indiens en Grande-Bretagne, etc.). Comment la T.A.L. peut-elle prendre en compte les difficultés de traduction liées à ces problèmes d'adaptation? Il semble qu'un apport d'informations soit indispensable pour définir au mieux ce processus de traduction. C'est dans ce but que nous exposons des travaux sur l'adaptation verbale qui implique une visée communicationnelle en L2.

\section{Exploitation de la langue cible par le sujet psychologique : modélisation du dialogue et visée communicationnelle en L2}

\subsection{Modélisation du dialogue : conditions de l'adaptation verbale à l'interlocuteur selon l'origine culturelle}

Le discours dialogique implique pour le moins une double référenciation, celle de l'autre à qui l'on parle et celle de la négociation du code commun pour l'objet dont on parle. L'adaptation verbale à l'interlo- 
cuteur consiste en une certaine quantité de reformulations afin d'atteindre cette double référenciation de façon la plus précise possible (Vivier, 1997). Nous référant ainsi à ces trois pôles de l'interlocution: locuteur-interlocuteur-objet de référenciation ( $c f$. Bühler, in Todorov, 1972), nous avons pris comme objectif d'étudier l'ajustement du discours en français-seconde langue. Les indices que nous retenons sont notamment les interférences et les reformulations, lesquels constituent d'excellents indices pour étudier l'ambiguïté référentielle. Nous postulons que celle-ci ne peut qu'être élevée, puisque deux codes linguistiques se surajoutent. Tout se passe comme si les jeux de langage (François F., 1985) se multipliant par deux, il existait un double niveau d'interprétation des intentions des partenaires.

\subsubsection{Régulation de la communication au sein de la famille (milieu naturel) : approche psychoculturelle}

Notre étude a porté sur des interactions dans un contexte familial. Ce type de travaux peut en effet éclairer sur la complexité du fonctionnement de la référenciation culturelle dans le passage d'une langue à l'autre, complexité qui varie selon les types de langues. Nous savons toutefois peu de choses au niveau des régulations de la communication au sein des familles; encore moins avec deux langues en présence. Cependant quelques études récentes apportent quelques éclaircissements.

Ainsi, Hausendorf (1993) s'intéresse aux aspects de l'étayage de l'auditeur adulte dans l'interaction avec des narrateurs enfants, ceux-ci étant âgés de 5, 7 et 14 ans. Il s'interroge sur le rôle de l'interaction pour l'acquisition de compétences discursives. De façon systématique, l'adulte se manifeste sous la forme $d^{\prime}$ "obligations", telle que l'injonction explicite (ex: "Allez raconte, qu'est-ce qui s'est passé là ? "), qui peut proposer soit une obligation globale (celle de raconter par exemple), soit une obligation globale implicite (caractère événementiel de ce qui s'est passé), ce qui indique pour l'enfant un passage digne d'être raconté. Avec (ou non) un silence, on provoque ainsi la narration. Comment l'enfant satisfait-il à cette obligation? Cela dépend de l'âge et du stade développemental du narrateur; cependant les analyses de cet auteur montrent que les plus jeunes ( 5 ans) "ne semblent percevoir les obligations globales que quand le partenaire adulte les établit explicitement». "Le traitement des obligations globales semble donc être une dimension spécifique au sein du développement de la compétence discursive ".

Hausendorf et Quasthoff (1989) précisent que dans la dyade adulteenfant, l'adulte doit maintenir un surplus d'activité pour entretenir la régulation de la narration, ce qui n'a pas lieu d'être entre des enfants d'âges proches, où les efforts respectifs sont comparables. Si l'enfant ne 
joue pas le jeu de la dépendance conditionnelle établie par l'adulte, celuici peut:

- y satisfaire lui-même, en conservant son rôle d'auditeur et en respectant ainsi les répartitions des rôles (auditeur/narrateur). Cela peut servir d'introduction à une meilleure obligation globale (un exemple est mis en œuvre).

- traiter les éléments narratifs de l'enfant «comme si » ils répondaient de manière suffisante aux conditions globales, ou traiter la réponse de l'enfant comme s'il s'agissait d'un événement extraordinaire ou suffisamment intéressant pour être raconté.

- localiser le cadre du récit; de façon différentielle, cette localisation est liée aux attributions de compétence par rapport à la classe d'âge. Ainsi, cette «localisation d'obligations globales" précisée par l'adulte n'intervient de manière systématique que pour des enfants de 5 ans. De la part des enfants de 5 ans, l'adulte attend un «rapport», de la part de ceux de 7 , 10 et 14 ans, une "narration scénique » (cf. Hausendorf et Quasthoff, 1989). De cette manière, l'adulte ajuste et anticipe ses activités en fonction de ce qu'il attribue à l'enfant, "en établissant des obligations spécifiques, un niveau de compétence spécifique » (Hausendorf, 1993). Il est exceptionnel, avec les narrateurs de 5 ans, que l'adulte réponde immédiatement à sa propre obligation globale; de même manière, un échec de l'interaction du fait de l'incapacité à satisfaire cette obligation est rare. Ce type de médiation est résumé en termes de prise en charge, de localisation, d'explicitation, et de traitement «comme si ». Observant le développement de la compétence narrative de l'enfant grâce à l'étayage, de nombreux auteurs s'appuient sur les travaux empiriques de Bruner et de ses collaborateurs, ainsi que sur Vygotsky, redécouvert dans les années 80, et son concept de «zone proximale de développement» (Vygotsky, 1978). En effet, ces études montrent l'importance des interactions adulte-enfant autour de la solution commune d'une tâche à laquelle l'enfant seul aurait été incapable de répondre. Bruner et Schaffer (1977) montrent qu'un des aspects fondamentaux de l'étayage est que le partenaire adulte s'efforce de diriger l'attention de l'enfant sur des objets, des faits, des activités particulières. Ces formats d'interaction de Bruner entre la mère et le jeune enfant se retrouvent sous la forme d'obligations entre enfant et adulte pour Hausendorf. Étant donné que nous intervenons dans notre démarche expérimentale au sein même du foyer de chaque famille, nous pensons pouvoir prélever ne serait-ce qu'un petit échantillon de routines interactives familiales. Il va de soi que la nature de la tâche expérimentale imposée peut modifier ces routines. Cependant nous pensons que les habitudes langagières reprendront le dessus après les premiers échanges généraux, 
et que l'implication dans la tâche ainsi que dans ces échanges face aux interlocuteurs habituels ne pourra que replacer l'enfant dans son cadre habituel de référenciation. Nous avons entrevu les possibilités émises par Hausendorf et coll. sur les contenus de l'interaction enfant-adulte en situation de narration : on peut relier ces échanges avec des dépendances de locus de contrôle externes (peu d'explicitations) et internes (explicitations). Voyons maintenant ce qu'il advient dans le cadre d'une interaction enfant-adulte familier, c'est-à-dire le père ou la mère: dans ce cas, l'interaction devient oblique et non verticale, à cause de la charge affective représentée au niveau de la relation enfants-parents. De la "grammaire naturelle » de Chomsky (1965), à la «mère omnisciente » de Schatz (1981), on peut prendre du recul. Tout d'abord l'adulte ne rend pas toujours le langage plus accessible à l'enfant: l'évitement de l'usage des pronoms personnels dans le discours au tout jeune enfant en est un exemple (Durkin, 1987, explique que cela complique les données pour l'enfant). Imaginons un instant des enfants confrontés à une langue maternelle, le cambodgien, où les données linguistiques sont extrêmement simplifiées avec absence de pronom personnel, de conjugaison, de genre et de nombre: ils sont alors d'emblée dans la situation de difficultés évoquée par Durkin. Ils doivent ensuite opérer la différenciation entre L1 et L2, de façon métalinguistique, en acceptant que dans la L1, on continue à opérer cette omission, et qu'en français- $\mathrm{L} 2$, on doit tout au contraire préciser chaque pronom personnel. Les interlocuteurs adultes français tolèreront de façon implicite, au regard de leurs propres conduites avec les jeunes enfants, ce type d'erreurs. On conçoit qu'à l'âge de 4 ans, un enfant cambodgien n'utilise que très peu de pronoms personnels en français, ce que nous allons vérifier ( $c f$. observation en III); cela s'expliquerait relativement aisément. Cela ne va-t-il pas prolonger la difficulté, ou la confusion dans l'esprit de l'enfant? La question de la médiation se pose plus que jamais, et les réponses apportées par l'étude du bilinguisme peuvent permettre d'éclairer l'étude de l'appropriation du langage.

Nous sommes dans l'obligation de faire dès à présent une démarche de type interculturel (et psychoculturelle) pour étudier ces relations obliques et ces pratiques éducatives, en nous appuyant tout de même sur les derniers travaux épistémologiques concernant les grandes lignes de développement pour ce qui concerne les cultures indo-européennes, dont le français fait partie. Dans certaines cultures, et c'est le cas de la culture cambodgienne, on s'adresse rarement verbalement à l'enfant tant que celui-ci ne sait pas parler (Ochs, 1985 ; Heath, 1983); dans d'autres, c'est le cas des cultures latino-américaines et françaises, on s'adresse à l'enfant avant que celui-ci ne parle; cela peut être efficace, ou non (par incitation à la simple imitation, par exemple). Ce qui semble universel est le changement proso- 
dique effectué par les parents (et les adultes en général) à l'égard des enfants. Nous ne disposons pas de données suffisantes à travers la littérature scientifique pour élucider le moment et la façon dont l'enfant commence à comprendre ce qui se passe autour de lui. Par contre, le facteur pragmatique entre en jeu dans le bain linguistique : l'enfant de 24 à 36 mois comprend et intervient dans les interactions entre mère et fratrie (Dunn et Schatz, 1989). Lieven et Pine évoquent trois universaux de développement:

1) la faculté de créer des jeux avec les mots par l'imitation répétitive.

2) la faculté de retrouver, par la mémoire, le lien entre un énoncé imparfaitement compris et la pragmatique de la situation au cours de laquelle il a été initialement produit (Snow, $1983: 3$ ).

3) une sensibilité particulière à la fréquence et à la «routinisation » d'énoncés et d'événements (Lieven, 1987).

Les enfants imitent des énoncés qui ont un rapport avec une situation répétée de dialogue routinier. Fillmore (1979) fait état de stratégies très similaires de la part d'enfants apprenant une langue étrangère (en situation scolaire). L'enfant gardé par sa mère apprend rapidement à répéter ce que la mère dit, celle-ci reformule jusqu'à ce que l'enfant réagisse et intègre plus ou moins correctement un énoncé ou un mot. Prenons un recul interculturel et voyons ce que cela peut apporter tant chez les hispanophones que chez les cambodgianophones. Chez les hispanophones, C. Perdue (1986) montre l'importance de la familiarité du référent, qui «fonde par exemple l'usage contrasté, Article défini + Nom/Article indéfini + Nom, ou le recours aux pronoms anaphoriques. Ces usages, on a pu le constater, sont transférés très tôt par les hispanophones". Mais pour revenir plus précisément à l'interaction oblique, nous devons préciser que les échanges et les pratiques éducatives latino-américaines sont quasisimilaires entre les cultures française et latino-américaine. Elles font partie des cultures à stimulation orale pré-linguistique (Ochs, 1985, et Heath, 1983), et ont été influencées par les mêmes modèles. Le jeune enfant est stimulé au niveau verbal de façon précoce, on attend de lui compréhension et répétition de mots et de phrases. On accepte l'expression de sa personnalité. Tandis qu'en France on aura tendance à lui parler à la troisième personne, en Amérique Latine, plus spécifiquement au Chili, on utilisera souvent le «usted » (vous) qui correspond à cette troisième personne du singulier. L'adulte personnalise ainsi l'enfant, le place de façon exagérée comme "une autre personne»; il faut croire que le «tu» ne suffit pas, est trop banal et que cette attitude rejoint les stratégies utilisées par l'adulte au niveau de la narration: il exagère un événement pour le rendre exceptionnel, digne d'être raconté; il semble que la même hypothèse peut être faite au niveau des pronoms personnels, c'est-à-dire que 
l'adulte pense ainsi attirer l'attention de l'enfant (ce qui n'est pas forcément une bonne idée selon Durkin, 1987). En fait, cela sert plus l'adulte que l'enfant, car cela lui donne l'impression (ou l'aide à le persuader) que l'enfant est déjà sıne personne, qui comprend ce qu'on lui dit. Il y aurait donc un surinvestissement de la personne, au même titre que le surintérêt pour la narration. Pour les cultures françaises et latino-américaines, nous pouvons nous référer aux travaux de Miguel Siguán Soler (1977) qui insiste sur le rôle important de la prosodie dans l'apprentissage du verbal, ainsi que sur la référence de l'adulte à "la réalité concrète immédiate: les personnes et les objets environnants et leurs qualités sensibles, les sentiments manifestes, les actions actuelles ou imminentes. L'enfant peut expérimenter tout (ou presque) ce que dit l'adulte ". Le contenu des échanges est simplifié et "hautement réitératif ", avec de nombreuses désignations.

Les parents des petits Cambodgiens adressent peu la parole aux enfants, puisque leur culture fait partie de celles qui ne cherchent à parler aux enfants que lorsque ceux-ci commencent à le faire d'euxmêmes. Il apparaît que les interactions au sein de la fratrie sont primordiales pour l'apprentissage de la L1. L'importance de l'âge, les fonctions de respect établies vis-à-vis des aînés va faire se prolonger au fil des années cet état de passivité vis-à-vis des parents, du père en particulier, lequel doit se comporter de façon très réglée.

\subsubsection{Relations psycholinguistiques: quelques observations} sur les routines interactives culturelles et la $L 2$

\section{- entre enfants et adultes}

Il est intéressant de noter que peu d'enfants cambodgiens issus de cette génération exilée parlent leur L1. Ils la perdent en grandissant, ou ne disposent que d'un vocabulaire très limité. Suite à nos entretiens avec des traducteurs cambodgiens et à nos observations des parents, on note tout d'abord la confirmation de l'existence d'un manque de communication entre parents et enfants. La communication est très ritualisée selon l'âge, le rôle, le statut de l'interlocuteur; elle est très hiérarchisée, l'âge et le statut passant avant le sexe. De plus, le cambodgien fait partie des langues dont la fonction est avant tout celle de l'instrument de communication; par conséquent il y a peu de nécessité et de valeur à communiquer avec des enfants très jeunes. Le non-verbal, les sourires et les gestes y suffisent amplement.

Si l'on ajoute une tendance à une formulation descriptive plutôt que narrative quelle que soit la langue utilisée (L1 ou L2), on peut imaginer que le taux de connecteurs permettant une fluidité verbale sera certainement peu élevé. L'importance des mots résidant plus dans leur contenu 
que dans leur forme, on suppose que les interférences risquent d'être très bien tolérées et non corrigées. Le manque de précision en français doit se trouver aggravé du fait de ces routines interactives: le mode spécifique d'énonciation cambodgien préconise des précisions ultérieures selon le contexte et l'interlocuteur. Les adultes cambodgiens reconnaissent euxmêmes leur grande difficulté - ils parlent même de handicap - en français oral, surtout au niveau lexical et grammatical. Dans leurs mots à eux, on entrevoit très vite le problème d'une pensée différenciée en cambodgien, opposée à la pensée française. Certains traducteurs parlent $d^{\prime}$ " intelligence française " et $d^{\prime}$ ' intelligence cambodgienne », on se trouve clairement devant des problèmes de cognition en matière de méthodes d'apprentissage ou de travaux de traduction. Si ces traducteurs parlent "d'intelligences » différentes, ou de manières de penser, de raisonner différentes, on imagine les difficultés de communication - qu'évoquent d'ailleurs les parents - qui s'accumulent lorsque les enfants grandissent, le français devenant la langue parlée le plus souvent; le registre de pensée, de raisonnement se fait donc dans cette langue. Un fossé peut ainsi se creuser entre les générations, éloignant parents et enfants au niveau production orale et compréhension. C'est ainsi que les aînés prennent le relais dans l'éducation des plus petits: on peut donc arriver à observer des enfants de 18 mois, petits derniers, parler directement la L2, alors que les prédécesseurs dans la fratrie ne parlaient, eux, que la L1. Il y a donc une adaptation des parents aux nécessités, aux contraintes du bain linguistique, du pays d'accueil français, qui va jusqu'à l'abandon de l'éducation linguistique en L1 dans certaines familles (du moins au niveau de la production en L1). Le problème réside alors dans un risque de dévalorisation des enfants de la langue et du système culturel d'origine.

Dans les interactions adultes-enfants, le support écrit n'est pas utilisé, puisque le fait d'écrire est presque une chose impossible pour la plupart des Cambodgiens. Le rapport serait quasiment le même que celui qu'entretient un Français avec le latin. Il va de soi que, comme pour l'arabe classique, il y a une différence entre écrit classique cambodgien et écrit «oral ", c'est-à-dire traduction littérale du langage parlé, qui reste accessible à certains. Mais il n'y a pas d'utilisation de supports écrits comme le livre d'images, ou les contes pour enfants, qui sont difficiles d'accès et peu répandus. On ne peut donc compter que sur les routines interactives pour la conservation et la transmission de la L1, et pour l'acquisition du français. Elles joueront donc un rôle crucial dans le rapport à la L1 et à la L2.

\section{- Routines interactives entre enfants}

Dans la majorité des cas observés, les routines interactives entre enfants s'effectuent en français en grande majorité, le recours au cambodgien ne 
servant que pour traduire des mots ou des énoncés qui ne sont pas compris, ou pour reprendre des énoncés affectifs, donc liés à des émotions: plaisir, jeu, colère, disputes, réprimandes. Le cambodgien sert aussi, comme pour beaucoup de bilingues, à s'isoler d'autres interlocuteurs français pour exprimer des choses de façon complice entre frères et sœurs (mais aussi entre amis ou enfants et parents). Par contre, le français est très descriptif et souvent difficile à comprendre pour un natif, étant donné l'influence du bain linguistique d'origine qui se perçoit dans les réponses, que ce soit au niveau prosodique, syntaxique, ou au niveau de la construction des phrases. L'importance du non-verbal est surprenante avec les tout-petits, mais aussi entre enfants plus âgés (7-8 ans): les silences peuvent être longs lorsqu'ils s'affairent sur une tâche. Les familles étant souvent nombreuses, il se forme des regroupements par âge ou par centre d'intérêt (les filles autour des poupées, les garçons autour des voitures). Les enfants les plus âgés imposent un respect et ne se mélangent pas pour des jeux futiles (qu'il s'agisse de jeux manuels ou de jeu verbaux) avec leurs cadets. Mais les aînés, qu'ils soient garçons ou filles, maternent souvent en cambodgien les tout-petits avec quelques consignes répétitives, rapides ou précises (exception faite pour le cinquième ou le sixième enfant qui se trouve soudain entraîné dans des routines interactives françaises, l'aîné s'étant aperçu de son travail de pédagogue et de traducteur au fil du temps).

- Communication entre natifs et apprenants (communication verticale et horizontale)

Nous avons parlé de l'importance des routines interactives au sein de la famille. Mais il est un autre fait aussi primordial qui est le rôle de l'appréciation par le natif de la production verbale du sujet. Cette appréciation sera déterminante, tout aussi bien pour les enfants que pour les parents qui auront vécu un certain type de régulation de la communication avec des interlocuteurs français: ainsi un certain type de réaction sera déterminé au sein des futures interlocutions puisqu'il y aura eu apprentissage avec une plus ou moins grande facilitation, déclenchant une plus ou moins grande motivation, ainsi qu'une plus ou moins grande production, correcte ou semi-correcte, avec ces mêmes interlocuteurs français. Ainsi, des démarches spécifiques seront adoptées dans les "stratégies " enseignées par les parents aux enfants, ou tout au moins dans les rapports à la L2 qu'ils véhiculeront et inculqueront à leurs enfants. Comme le résume B. Py (1993), l'apprenant a son territoire (contexte linguistique dans lequel se déroule l'acquisition), avec son système (de connaissances linguistiques, ou interlangue), sa norme (normes de la L2) et sa tâche (toute activité communicative en L2); parmi ces trois éléments principaux, le "système» joue un rôle fondamental; particulier à chaque langue, il 
contient des éléments universaux, mais aussi, en matière de bilinguisme (Grosjean et Py, 1991) des zones très instables qui subissent des modifications. Py réfute l'idée que la tâche soit plus importante que le rôle de l'interaction (ce qui est trop souvent démontré dans les recherches à visées pédagogiques, qui ne prennent pas assez en compte la correction grammaticale). «L'interaction n'est pas seulement une locomotive qui tirerait la grammaire derrière elle; elle fournit une partie au moins des schèmes qui appellent et rendent possible tout à la fois la construction de microsystèmes grammaticaux. D'autre part, la manière dont l'alloglotte respecte ou enfreint les normes de ses interlocuteurs joue un rôle dans le déroulement même de la tâche, et, au-delà, dans la construction des connaissances " (Py, 1993). Cette norme peut être souple ou non, elle est dépendante de la L2 ainsi que du natif en L2 et de sa "zone de tolérance" ou d'écoute. Pour $q u$ 'il y ait régulation de la communication entre natif et apprenant, il faut que le premier utilise des normes du «juste vs faux» (le natif doit déterminer à partir de quel seuil d'erreurs il admettra un énoncé comme correct ou incorrect en situation orale), qui vont amener une série de démarches communicatives, telles que l'injonction, une certaine prosodie amenant à une reformulation; des acceptations correctrices ou non, en résumé tout un système dépendant de ces normes alliées à la personnalité du natif : celui-ci impose ou non une situation didactique, qui peut s'avérer trop lourde (corrections systématiques, mésentente simulée ou vérifiée, etc.) ce qui peut empêcher la progression de l'apprenant; l'inverse peut être tout aussi invalidant pour la L2 ; une incitation de correction trop superficielle peut faire croire à l'apprenant qu'il est dans la bonne voie et entraîner des erreurs chroniques. Il est donc nécessaire que ces situations d'interlocution entre natifs et apprenants soient régulières et diversifiées (dans des contextes et avec des interlocuteurs natifs variés) pour qu'une auto-correction surgisse et que la communication soit riche. Tout dépendra de l'identité et de la personnalité du sujet: l'apprenant extraverti et loquace diversifiera ses connaissances lexicales, mais peut demeurer à un niveau idiosyncrasique. Qu'il soit enfant ou adulte, ce sont les fonctions d' "étayage » et de "tutelle» dont parle Bruner qui vont diriger et structurer les échanges. Le natif prend alors le rôle d'expert et permet ainsi à l'apprenant de réaliser une performance qui aurait été impossible sans son aide. Hudelot (1992) précise qu'en plus de certaines informations nécessaires (comme des propositions lexicales), l'expert s'engage sur les «plans cognitif, affectif et relationnel ».

\section{- natifs et hispanophones}

Nous avons vu que selon des auteurs comme Cammarota, Giacobbe (1986), les ponts pouvant être effectués entre L1 et L2 lorsque celles-ci sont 
proches permettent des stratégies d'apprentissage ; ces stratégies sont permises après une conscientisation de ces liens possibles; plus cette conscientisation est précoce, plus l'apprentissage serait facilité.

\section{- natifs et Cambodgiens}

Nous avons déjà évoqué les codes culturels asiatiques, si différents des codes français. Il semble qu'ils soient aux antipodes les uns par rapport aux autres, et les logiques énonciatives, cognitives et communicationnelles possèdent tous les éléments nécessaires pour que natifs français et Cambodgiens ne se comprennent guère.

\subsection{Référenciation appuyée sur des registres de communication différents}

Voici une illustration de registre interactif cambodgien fonctionnant sur la régulation communicationnelle par la répétition du dernier mot, au lieu d'une régulation phatique, par exemple.

E.8. Et boulanzerie;

O.9. Boulanzerie;

E.9. Et...

0.10. Une' gare;

E.10. Gare;

o.11. Voiture;

E.11. Voiture;

O.12. Camion;

E.12. Camion ;

0.13. Fuh... Fuh... Euh... Vache;

E.1.3. Vache;

O.14. Euh... Euh... Euh ...

E.14. Chevre;

0.15. Une' chirrere; coiffeur;

Remarquons qu'entre ces deux fillettes cambodgiennes de 8 (codée $\mathrm{O}$ ) et 6 (codée E) ans, les échanges sont courts, comme en L1. L'énumération renforce l'absence de déterminants, semblant faire resurgir le système de catégorisation cambodgien (cognition cambodgienne). L'utilisation exacte du déterminant pour le mot "chèvre " laisse penser qu'il s'agit bien du registre cambodgien plaqué sur le français et non pas d'une méconnaissance totale du genre ou de la nécessité de placer un déterminant en français. En début d'interlocution, les premières phrases comprennent des déterminants parfois corrects. Dès qu'il y a énumération, on observe une omission des déterminants. L'une comme l'autre des deux enfants répètent le dernier mot énoncé. L'énumération (donc le système de catégorisation cognitive), entraîne systématiquement la répétition du même mot, ou de plusieurs, et l'absence de déterminants, comme par écholalie. On retrouve ce fonctionnement avec les parents. Au niveau des 
interférences, les omissions de déterminants sont plus massives en tâche descriptive qu'en tâche narrative. Au niveau du type d'interférence, ce sont les omissions qui priment sur toute autre interférence (comme l'inversion masculin-féminin, par exemple).

\subsection{Dialogue inhibé par une communication indirecte: exemple de dialogue sorti du contexte extralinguistique de l'interlocution (quid des intentions des interlocuteurs ?)}

Dialogue entre deux enfants cambodgiens de $4\left(N^{\circ} 4\right)$ et 6 ans $\left(N^{\circ} 1\right)$ :

1.10. Ben attends, lui qui fait rien!

4.9. C'est lui!

1.11. Est fainéant lui.

4.10. Agnès; d'ha-bitude parce qu'il est en train de le faire exprès là. Regarde.

Sans le contexte extralinguistique, et sans connaissance du fonctionnement de la communication culturelle implicite en question, il n'est pas possible de comprendre cet échange en français/L2. Les deux enfants s'adressent directement à leur frère (en le regardant) qui est apparu dans le fond de la pièce, puis à l'adulte (Agnès) :

La traduction en serait :

1.10. Ben attends, toi qui ne fais rien!

4.9. Toi là-bas! (ou: oui, toi!)

1.11. Tu es fainéant toi.

4.10. Agnès; d'habitude, il nous aurait aidés, mais là il est en train de le faire exprès (de ne pas nous aider alors que c'est son rôle d'aîné). Regarde (interviens, toi qui est plus âgée).

\subsection{Les pronoms personnels}

Les pronoms personnels ne sont pas marqués en cambodgien, le mode de conversation entre deux personnes ne peut donc se dérouler de la même manière qu'en français. Peu de choses sont définies en début de dialogue, puis, selon le contexte, l'interlocuteur, son statut et son âge par rapport à ceux du locuteur, selon la situation dans laquelle ils se trouvent, des précisions seront apportées. L'échange sera fondamentalement construit à deux. L'adaptation des Cambodgiens en France est perturbée par cette forme culturelle de construction de l'échange; la structure de la langue maternelle est souvent plaquée sur le français, d'où un non-respect de la grammaire, l'absence de pronoms personnels, l'absence de précisions en général. Les routines interactives de ce type de langues inhibent l'utilisation des pronoms personnels, la catégorisation grammaticale en français et majorent les détails spatiaux. Une approche interculturelle et dialogique est nécessaire pour analyser ce bilinguisme formé de deux langues éloignées. De nombreuses langues fonctionnent avec ce mode de 
personnalisation spatiale; or ce mode de catégorisation est peu étudié car les travaux sont teintés d'interprétation interne occidentale ne prenant pas en compte ces considérations (par exemple, les anciens dictionnaires khmer/français font état de la traduction des pronoms personnels alors que dans l'usage on ne les pratique pas).

\section{Un exemple d'observation armée}

\subsection{Les conditions d'observation}

Nous avons pris en compte les exigences de notre approche interculturelle et psycholinguistique : nous avons défini une situation d'observation qui tente de concilier les propriétés écologiques des contextes de la communication et les contraintes que suppose la rigueur d'une observation instrumentale. Pour entrevoir l'impact différencié des routines interactives culturelles, il était indispensable d'enregistrer les enfants (sur magnétophone), en situation de dialogue d'une part, en milieu naturel familier (à domicile) d'autre part : d'où la pertinence d'une analyse de discours dialogique accompagnée d'une méthodologie écologique. Nous aurions souhaité une caméra invisible à l'appui, ce qui n'était pas envisageable dans une telle étude sur le terrain, en particulier auprès de populations de réfugiés très difficile d'accès. Des conditions standard de passation permettraient en outre une analyse statistique complémentaire. Ces conditions sont les suivantes: deux groupes d'enfants, un groupe de 10 petits Cambodgiens âgés de 4 à 9 ans et un groupe contrôle de 10 enfants latinoaméricains hispanophones, dont les parents ne parlent que la langue maternelle à domicile (le français n'est donc utilisé que de façon institutionnelle ou de façon proximale avec le voisinage) ont trois types d'interlocuteurs: un parent, un enfant de la fratrie, et l'expérimentatrice adulte française; deux types de tâches sont prétextes de dialogues, l'une descriptive-contraignante (commentaire d'un livre d'images en temps limité), l'autre narrative-permissive (récit à partir d'une image de puzzle); deux situations locatives étaient proposées, à domicile, et dans une Maison de Quartier (variable témoin). L'une des deux tâches était censée représenter une difficulté linguistique rappelant la langue source pour le groupe d'enfants cambodgiens: une tâche contraignante et descriptive pouvait rappeler à ces enfants le système linguistique d'origine étant donné que la description est tout de même un élément important dans la langue cambodgienne tandis que la tâche narrative et libre était censée déclencher la production ou les interférences en L1 chez les hispanophones. 


\subsubsection{Hypothèses}

Hypothèse générale:

À partir du postulat de typologie différentielle des langues, les difficultés d'acquisition du français comme langue seconde seront plus importantes si la langue maternelle est de type implicite (comme le cambodgien): une adaptation cognitive sera alors nécessaire (nouvelles représentations, nouvelles catégorisations, un registre de pensée et de communication différent), contrairement à un bilinguisme formé de deux langues explicites (espagnol/ français).

Quelques hypothèses opérationnelles:

H.O.1 : selon le type de langue maternelle et selon l'existence de routines interactives variera la manière dont les enfants utiliseront les pronoms personnels dans leur discours en langue seconde.

H.O.2 : La complexité narrative (évoquée par les connecteurs et les reprises anaphoriques) provoquée par une tâche permissive libre n'apparaît que tardivement en français/L2 chez les enfants cambodgiens au niveau développemental (vers 8 et 9 ans); elle sera plus précoce chez les enfants hispanophones du fait des ponts linguistiques existant entre les deux langues 1 et 2 (recul métalinguistique facilité).

\subsubsection{Résultats}

Analyse Factorielle des Correspondances Multiples:

Récapitulatif pour la personnalisation:

Axe $1(28 \%)$

Utilisation de la langue maternelle: elle est maximale chez les hispanophones, quels que soient la tâche, l'âge, l'interlocuteur, la situation locative, alors qu'elle est minimale chez les cambodgianophones.

L'opposition est frappante avec le partenaire parent: il y a codeswitching d'un côté, mais pas de l'autre.

Cette utilisation du français et de la langue maternelle est donc très différenciée selon la L1, quel que soit l'âge pour les deux groupes. De plus, une utilisation forte de la L1 n'empêche ni la correction ni la production qui sont maximales en français chez les hispanophones, alors que l'utilisation totale du français n'entraîne que correction et production minimales chez les Cambodgiens (en particulier auprès de l'interlocuteur familier).

Axe $2(26 \%)$ :

Perception de la tâche descriptive (livre) selon l'âge, chez les hispanophones (en majorité) : correction et production sont maximales quel que 
soit l'interlocuteur chez les hispanophones de 8-9 ans, en opposition à des corrections et productions minimales avec l'adulte français (et une production moyenne avec la fratrie) pour des hispanophones de 4 à 6 ans (les plus jeunes) et un Cambodgien de 8 ans (ainsi que des Cambodgiens d'âges variés mais à contributions plus faibles).

Par rapport à la tâche descriptive, la tâche narrative ne favorise que l'échange avec l'adulte (familier et non-familier) chez les hispanophones de 8-9 ans alors qu'il l'entrave avec l'adulte français et la fratrie pour les jeunes hispanophones de 4 et 6 ans et les Cambodgiens d'âges variés, jusqu'à l'âge de 8 ans.

\section{Synthèse:}

Au niveau quantitatif, une analyse factorielle des correspondances multiples confirme un premier aspect différentiel selon la culture dans l'échange dialogique: d'une part, une grande utilisation de code-switching (alternance de langues) par les hispanophones, facilitant la correction et la production des indices (personnalisation, utilisation de catégories grammaticales telles que les déterminants, les pronoms personnels, les reprises anaphoriques) en français - comme en langue maternelle d'ailleurs. D'autre part, il n'y a pas ou peu d'utilisation de code-switching par les enfants cambodgiens, qui utilisent principalement le français; par contre, la correction et la production des indices précités sont minimales.

Au niveau qualitatif, l'acquisition des connecteurs en L2 ne pose pas de problèmes chez les hispanophones: dès 4 ans, la liste de ces connecteurs est variée. Inversement, ceux-ci sont rares chez les sujets cambodgiens (et cela s'explique puisqu'ils n'en utilisent que peu en L1; c'est l'ordre syntaxique qui semble important) et n'interviennent que tard dans le développement (vers 9 ans). Les omissions de pronoms personnels constituant des pratiques courantes, la reprise anaphorique s'effectue rarement en termes de pronoms personnels. Les seules constructions anaphoriques rencontrées dans les échanges verbaux se font avec de courtes relatives, et ceci seulement chez les plus âgés (9 ans). Cette omission quasi systématique de connecteurs, de reprises anaphoriques et la pauvreté des relatives semble démontrer une absence de narration; on ne perçoit celle-ci que si on la cherche dans la construction syntaxique, à la lumière des routines d'échanges cambodgiennes. On comprendra que certains noms remplaceront des verbes conjugués lorsque des déictiques ou des prépositions les accompagnent (exemple D); on se rend compte que l'action précède la fonction, l'état, la définition de Soi (exemple A); que la cause se place dans un second énoncé (exemple B); qu'un mot-clé (substantif ou connecteur) en fin de prise de parole peut relier tous les énoncés précédents (exemple C et E. Cf. illustrations ci-dessous). 
Exemple A:

4.35. Il met (de l'eau)... Il fait du pompier. C'est pompier.

Exemple $B$ :

4.36. Y met de l'eau. Il y a le feu.

Exemple $C$ :

Un tigre.

P.2. Et ça?

1.2. Un loup. Un chien. Un serpent et puis le bonhomme.

(le bonhomme agit sur tous les autres animaux, est le personnage actif et principal)

Exemple D:

Non, pour toi c'est là.

(au lieu de: "Mets-le là »)

Exemple E:

C.12. Non, y'en avait des comme ça, y en avait des noirs. Noirs, je crois qu'y en a plus.

\subsubsection{Conclusion de l'observation}

Notre typologie différentielle des langues appuyée par l'utilisation ou non du code-switching dans l'interlangue permet de mieux cerner le processus d'acquisition d'une seconde langue: il s'agit là d'un modèle psycholinguistique d'apprentissage prenant en compte les facteurs culturels et communicationnels habituellement contournés ou considérés comme parasites. Nous cherchons actuellement à améliorer ce modèle en tenant compte des fonctions exogènes et référentielles dans les langues en présence, en particulier dans leur usage dialogique (avec les réactions et les reformulations des natifs).

\section{Discussion}

Nous pouvons nous référer à la typologie psycholinguistique des langues et aux routines interactives pour discerner le type de processus d'acquisition (son niveau de difficulté) et savoir si les types de construction de l'échange dialogique coïncident, s'il n'y a pas d'opposition implicite/explicite.

Le code-switching ne sert l'apprentissage d'une L2 que lorsqu'il repose sur deux langues de même type. Quant à la construction syntaxique, elle joue un rôle important dans la gestion du dialogue de type impliciteexterne: les implications psycholinguistiques et culturelles dans le processus d'apprentissage d'une langue seconde ne peuvent être ignorées, si l'on a affaire à deux types cognitifs de recours syntaxique intégrés dans la langue. Les Cambodgiens n'utilisent pas le code-switching, ils fonctionnent par le plaquage de la L1 sur la L2; ordre syntaxique, référenciation et mémoire de travail fonctionnent toujours en L1 et non en L2; il n'y a pas 
de reprise anaphorique des déterminants, des noms ou des pronoms personnels. Travailler sur des outils cognitifs issus de ces résultats permettrait d'améliorer l'apprentissage de la L2 ainsi que la traduction automatique.

\section{Conclusions à prendre en compte en TAL}

L'analyse de divers formats de référenciation en ce qui concerne le codage des propriétés linguistiques renvoie aux thématiques centrées sur la modélisation du langage et de la cognition culturelle et spatiale. La question se pose alors de savoir comment le sujet s'adapte aux contraintes de linéarisation imposées par le discours en L2. Il s'agit aussi de comprendre les rôles que peuvent jouer ici les divers formats de représentation. Les travaux présentés offrent la possibilité de mieux comprendre les caractéristiques du fonctionnement du sujet ou du groupe culturel, permettant ainsi d'envisager ce qu'il est susceptible de mettre en œuvre ou non dans une situation donnée; la TAL peut prendre en compte ces travaux en considérant, par exemple, l'importance de l'expression de la personnalisation: s'agit-il d'utiliser des pronoms personnels ou de renvoyer le sujet à sa localisation spatiale? Dans un dialogue bilingue à support informatisé, on peut observer une gêne considérable dans l'intercompréhension uniquement à cause de ce problème. Qu'il s'agisse d'un dialogue multiculturel ou monoculturel, la traduction (en L2, ou en autre registre langagier) prendra un temps plus long, plus cyclique pour les sujets de langues implicites ou à tendance externe (cf. aussi conclusions de Beauvois (1984) qui vont dans ce dernier sens); ceci devra être pris en considération pour les modélisations ciblant des réponses fermées de la part des sujets interrogés. 


\section{Références bibliographiques}

ABELSON, R.P., "Script processing in attitude formation and decision making ", in Carrol, J.S., Payne, W. (Eds), Cognition and social behavior, Hillsdale, N.J.: Erlbaum, 1976.

BEauvols, J.-L., La psychologie quotidienne, Paris, PUF, 1984.

Benveniste, E., Problemes de linguistique générale, Paris, Gallimard, Tome I, 1966.

BRICEHEATH, S., Ways with words, Cambridge, Cambridge University Press, 1983.

BRUNER, J.S., Acts of meaning, Harvard University Press, 1990.

BRUNER, J.S., in Schaffer, H.R. (Ed.), Studies in mother-infant interaction, London, Academic Press, pp. 271-289, 1977.

BüHLER, K., in Ducrot, O., et Todorov, T., Dictionnaire encyclopédique des sciences du langage, Paris, Seuil, 1972.

CAmmarota, M.-A., et GiACOBBE, J., "L'acquisition du lexique en français par des adultes hispanophones ", Langages, 84, pp. 65-78, 1986.

CHOMSKY, N., Aspects of the theory of syntax, Cambridge, MA: MIT Press, 1965.

DUNN, J.F., et SCHATZ, M., "Becoming a conversationalist despite (or because of) having a sibling », Child development, $60: 399-410,1989$.

FILLMORE, C.J., KEMPLER, D., WANG, W., Individual differences in language ability and language behavior, New-York, Academic press, 1979.

François, F., Hudelot, C., SABEau-JouAnNet, E., Conduites linguistiques chez le jeune enfant, Paris, PUF, 1984.

GrosjeAN, F., PY, B., "La restructuration d'une première langue : l'intégration de variantes de contact dans la compétences de migrants bilingues ", La linguistique, 27, 2, 35-60, 1991.

HALL, E.T., La dimension cachée, Paris, Point, 1972.

HAUSENDORF, H., "L'étayage dans l'interaction », Encrages, AILE, № 2, pp. 85-107, 1993.

Huteau, C., in Pémartin, D., Le test de locus de contrôle, Éditions et Applications Psychologiques, 1997.

LANGER, E.J., BLANK, A., CHANOWITZ, B., "The mindleness of ostensibly thoughtful action: The role of placebic information in interpersonal interaction ", Journal of Personality and Social Psychology, 36, 635-642, 1978.

LIEVEN, E.V.M., PINE, J.M., DRESNer BARNeS, H., "Individual differences in early vocabulary development: redefining the referential-expressive distinction », Journal of child language, $19: 287-310,1992$.

Morel, M.-A., DANON-Boileau, L., La deixis, Paris, PUF, 1990.

OCHS, C.E., "Variation and error: a sociolinguistic approach to language acquisition in Samoa ", in Slobin, D.J.,(Ed.), The crosslinguistic study of language acquisition, Vol. I, Hillsdale, N.J. Erlbaum, 783-838, 1985.

PERDue, C., VASSEUR, M.-T., in " L'acquisition du lexique en français par des adultes hispanophones ", Langages, 84, pp. 65-78, 1986.

PY, B., "Acquisition et interaction en langue étrangère ", Encrages, AILE, $\mathrm{N}^{\circ}$ 2, pp. 9-24, 1993.

QUASTHOFF, U.M., (1989), in Hausendorf, H., "L'étayage dans l'interaction", Encrages, AILE, $\mathrm{N}^{\circ} 2$, pp. 85-107, 1993.

SAlinas, A., "Processus d'acquisition du français comme langue seconde par deux groupes d'enfants: de langue espagnole et de langue cambodgienne. Les effets de variation de routines interactives", in Diálogos hispánicos, (Las Lenguas en la Europa Comunitaria "La adquisición de segundas lenguas y/o de lenguas extranjeras"), Amsterdam, Rodopi, N 13, 1994.

SAIINAS, A., "Acquisition d'une seconde langue dans le cas de deux bilinguismes très différenciés: une étude psycholinguistique et cognitive ", Psycholinguistics as a multidisciplinarily science, ed. by Silvana Contento, "Il Ponte Vecchio », Forti-Cesena, II, 1996. 
Salinas, A., "Processus d'apprentissage socio-cognitif et typologie différentielle des langues », Europe Plurilingue, $N^{\circ} 14,1998$.

Sicuan-SOlER, M., "De la communication gestuelle au langage verbal ", in Bronckart, J.P., Malrieu, P., Siguan-Soler, M., Sinclair de Zwart, H., Slama-Cazacu, T., Tabouret-Keller, A., La genèse de la parole, Paris, PUF, 1977.

SNOW, C.E., "The development of conversation between mothers and babies ", Journal of child language, $4: 1-22,1977$.

TOdorov, O., Ducrot, O., Dictionnaire encyclopédique des sciences du langage, Paris, Seuil, 1972.

VIVIER J., "Pour une psychologie du dialogue homme machine ", in Sabah G., Vivier J., Prince V., Pierrel J.M., Romary L., Nicolle A., Machine, langage et dialogue, Paris, L'harmattan, 1997.

VYGOTSKY, L.S., "Interaction between learning and development ", in Vygotsky, L.S., Mind and society, (John-Steiner, V., Scribner, S., Souberman, E., Eds.), Cambridge, Cambridge University Press, 79-91, 1978. 